\title{
Parameter identification for leaky aquifers using global optimization methods
}

\author{
Hund-Der Yeh,* Yu-Chung Lin and Yen-Chen Huang \\ Institute of Environmental Engineering, National Chiao Tung University, Hsinchu, Taiwan
}

\begin{abstract}
:
In the past, graphical or computer methods were usually employed to determine the aquifer parameters of the observed data obtained from field pumping tests. Since we employed the computer methods to determine the aquifer parameters, an analytical aquifer model was required to estimate the predicted drawdown. Following this, the gradient-type approach was used to solve the nonlinear least-squares equations to obtain the aquifer parameters. This paper proposes a novel approach based on a drawdown model and a global optimization method of simulated annealing (SA) or a genetic algorithm (GA) to determine the best-fit aquifer parameters for leaky aquifer systems. The aquifer parameters obtained from SA and the GA almost agree with those obtained from the extended Kalman filter and gradient-type method. Moreover, all results indicate that the SA and GA are robust and yield consistent results when dealing with the parameter identification problems. Copyright (C) 2006 John Wiley \& Sons, Ltd.
\end{abstract}

KEY WORDS groundwater; global optimization; leaky aquifer; parameter identification; simulated annealing; genetic algorithm

Received 12 April 2005; Accepted 19 December 2005

\section{INTRODUCTION}

Aquifer parameters are very important in site characterization. In a leaky aquifer system, the semi-previous beds (also known as the aquitard), although of very low permeability, may yield significant amounts of water to the adjacent pumped aquifer. Two mathematical models developed for dealing with leaky aquifers are chosen in this study. One is based on the assumption that the aquitard storage is negligible and the other takes into account aquitard storage.

Jacob (1946) developed a mathematical model for describing a non-steady radial flow toward a well in a leaky aquifer. The hydraulic head in the unpumped adjacent aquifer was assumed as a constant and the storage capacity of the aquitard was neglected. Hantush and Jacob (1955) described a non-steady radial flow toward a well in a fully penetrating leaky aquifer under a constant pumping rate. In their model, the aquitard is overlain by an unconfined aquifer, and the main aquifer is underlain by an impermeable bed. Their solution herein is termed the three-parameter model. Neuman and Witherspoon (1969) offered another solution, describing the drawdown of the lower and pumped aquifer in a hydrogeological system that is composed of two confined aquifers and one aquitard. Their solution eliminated the drawback of Hantush's small- and large-time solutions and was called the four-parameter model. Both the three-parameter and the four-parameter models were also mentioned in other researchers' work (Batu, 1946; Dawson and Istok, 1991).

\footnotetext{
* Correspondence to: Hund-Der Yeh, Institute of Environmental Engineering, National Chiao Tung University, Hsinchu, Taiwan. E-mail: hdyeh@mail.nctu.edu.tw
}

In the past, hydrogeologists have often determined the confined aquifer parameters, such as transmissivity $T$ and storage coefficient $S$, by using graphical methods, otherwise known as curve-fitting methods, to analyse the observed drawdown data. For a three-parameter leaky aquifer model, Hantush and Jacob's (1955) solution was employed to generate the type curves to determine $T, S$, and the leakage factor $B$. However, for the four-parameter leaky aquifer model, with an additional parameter accounting for the aquitard storativity, the graphical matching approach based on the Neuman and Witherspoon solution was practically impossible, as there will be several families of curve types. Besides, graphical approaches generally require data plotting work and individual judgement during the curve-fitting procedures, which may lead to further errors.

In addition to the graphical methods, the aquifer parameters can also be obtained using computer methods. Since we employed the computer methods, an analytical aquifer model was needed to estimate the predicted drawdown for the estimation of the aquifer parameters. The parameters obtained are usually determined using the least-squares approach by taking the derivative of the sum of square errors between the observed and predicted drawdowns with respect to the parameters. Then, a gradient-type approach, such as the non-linear least-squares and finite difference Newton's method (NLN), might be utilized to solve the non-linear least-squares equations for obtaining the aquifer parameters (Yeh, 1987; Yeh and Han, 1989). The NLN approach had the advantages of high accuracy and quick convergence for reasonable initial guesses when estimating the aquifer parameters via pumping test data. However, 
two disadvantages are involved when applying Newton's method (or gradient-type methods) for solving the nonlinear least-squares equations in which the target parameters are unknown. First, the problem of divergence might be inevitable if inappropriate initial guesses are made. Second, inaccurate results will occur if the partial derivative terms in the least-squares equations are poorly approximated. Recently, the extended Kalman filter (EKF) was successfully applied to aquifer parameter and water-table-related estimations. Yeh and Huang (2005) employed the EKF to identify the aquifer parameters in leaky aquifer systems with and without considering the storage effect in the aquitard. The results indicate that the EKF can be utilized to analyse the drawdown data even with white noise or temporally correlated noise. However, the problem of how to decide reasonable initial guesses for aquifer parameters and the error covariance matrix remains a tough challenge in using the EKF.

This study proposes an approach using simulated annealing (SA) or a genetic algorithm (GA) coupled with Hantush and Jacob's (1955) solution or Neuman and Witherspoon's (1969) solution to determine the best-fit aquifer parameters. In our approach, the aquifer drawdown equations are used to predict the drawdown based on the guess parameters and then SA or the GA determines the best-fit parameters in such a way to find the least sum of square errors between the observed and predicted drawdowns. Moreover, different key SA or GA parameters are assigned for studying the robustness of the proposed approach when applied to the problem of parameter identification.

All the results obtained from our approach indicate that the major advantages of using SA and GAs over other existing methods in parameter identification are that they are capable of randomly giving the initial guess values for aquifer parameters and obtaining optimal results without solving non-linear least-squares equations. In addition, the results also indicate that SA and GAs are very robust in handling the parameter identification problems given in the analyses.

\section{GLOBAL OPTIMIZATION METHODS}

Stochastic optimization techniques such as SA and GAs are famous global optimization methods. More recently, developments in the field of stochastic optimization have allowed the formulation of optimization problems as nonlinear mixed integer problems. Metropolis et al. (1953) first applied SA to a two-dimensional rigid-sphere system, and Kirkpatrick et al. (1983) employed it in solving large-scale combinatorial optimization problems. SA is a random search algorithm that allows, at least in theory or in probability, the obtaining of the global optimum of a function in any given domain. Goffe et al. (1994) presented results in global optimization of statistical functions with SA, and Zheng and Wang (1996) used the tabu search and SA to identify parameter structure. In groundwater modelling, the identification of an optimal flow or transport parameter that varies spatially should include both the values and the structure of the parameter. Cunha and Sousa (1999) used SA to minimize the capacity extension cost of a water distribution network. The solution set obtained from SA and non-linear programming (NLP) techniques for several medium-sized networks showed that SA did provide a better solution in general, in comparison with that obtained with the NLP techniques. The major advantage of SA lies in its property of using a descent strategy, but allowing random ascent moves to avoid possible trapping in a local optimum. The other advantages of SA are that its theory is very simple and its algorithm is easy to implement.

Holland (1975) developed GAs during the 1950s and 1960s. The basic concept of the GA is the theory of evolution. Using the population of guesses to solve the optimization problem significantly differs from those using a single guess point. The main idea is that the genetic information of a good solution spreads over the entire population. Thus, the best solution can be obtained by thoroughly combining the chromosomes in the population. Selection, crossover, mutation, and reproduction are the essential operators in a GA. The selection operator picks up the relatively fitter strings according to their objective function values. Then, the newborn trial solutions are generated from the relatively fitter string with a crossover operator. The main purpose of the mutation operator is to avoid the trial solution being trapped in a local region. In recent years, many researchers have employed GAs in various fields. McKinney and Lin (1994) presented a solution of groundwater management models using GAs, and Coley (1999) and Pham and Karaboga (2000) also indicated that GAs were a general stochastic evolutionary algorithm with a wide range of applicability to optimization problems and showed good performance. The following section gives a description of the theoretical frameworks of SA and GAs.

\section{Simulated annealing}

The concept of SA is based on an analogy to the physical annealing process. At the beginning of the process, the temperature is increased to enhance molecule mobility. Then, the temperature is slowly decreased to allow the molecules to form crystalline structures. When the temperature is high, the molecules show high activity and the crystalline configurations take various forms. If the temperature is cooled properly, then the crystalline configuration is in the most stable state; thus, the minimum energy level may be reached naturally.

At a given temperature, the probability distribution of system energy is determined by the Boltzmann probability (Pham and Karaboga, 2000)

$$
P(E) \propto \exp (-E / k T e)
$$

where $E$ is the system energy, $k$ is Boltzmann's constant, $T e$ is the temperature, and $P(E)$ is the occurrence probability. There exists a small probability that the system might have a high energy even at a low temperature. 
Therefore, the statistical distribution of energies allows the system to escape from a local minimum energy. This is the major reason why the solutions obtained from SA may not be trapped in a local optimum or obtain an ill solution. The Boltzmann probability is applied in the Metropolis criterion (Kirkpatrick et al., 1983), where $\Delta E$, the difference between the objective function values of the current optimal solution and the trial solution, takes the place of $E$ and $k=1$. The modified Boltzmann probability, the accepted probability of the trial solution, is given as

$$
P(\Delta E)=\exp (\Delta E / T e)
$$

As an iterative improvement method, an initial point $x$ is required to evaluate the objective function value $f(x)$. Let $x^{\prime}$ be the neighbour of $x$ and its objective function value is $f\left(x^{\prime}\right)$. The $x^{\prime}$ is given as

$$
x^{\prime}=x+\left(2 D_{1}-1\right) V M
$$

where $D_{1}$ is a random number between zero and one from a uniform distribution and $V M$ is the step length vector. In this study, $V M$ is automatically adjusted so that approximately half of all evaluations are accepted. In minimization problems, if $f\left(x^{\prime}\right)$ is smaller than $f(x)$, then the current optimal solution takes place with the trial solution $\left(x^{\prime}\right)$. If $f\left(x^{\prime}\right)$ is not smaller than $f(x)$, then one has to test the Metropolis criterion and generate another random number $D_{2}$ between zero and one from a uniform distribution. For solving the minimization problem, the Metropolis criterion is given as (Metropolis et al., 1953):

$$
\begin{aligned}
& P_{\mathrm{SA}}\{\text { accept } j\} \\
& \quad= \begin{cases}1 & \text { if } f(j) \leq f(i) \\
\exp \left(\frac{f(i)-f(j)}{T e}\right) & \text { if } f(j)>f(i)\end{cases}
\end{aligned}
$$

where $P_{\mathrm{SA}}$ is the acceptance probability of the trial solution, $f(i)$ and $f(j)$ are respectively the function value when $x=x_{i}$ and $x=a_{x}$, and $x_{i}$ and $a_{x}$ are respectively the current best solution and the neighbourhood trial solution of $x$. Here, $T e$, a control parameter, is usually the current temperature. If the random number $D_{2}$ is smaller than $P_{\mathrm{SA}}$, then the current solution also takes place with the trial solution. Otherwise, one must continue generating the trial solution within the neighbouring vicinity of the current solution.

Figure 1 illustrates the SA algorithm (Pham and Karaboga, 2000). The first step is to initialize the initial solution and set the initial solution to be the current optimal solution. The second step is to update the current optimal solution, if the trial solution generated from the initial solution within the boundary is better than the current optimal solution or if the trial solution satisfies the Metropolis criterion; otherwise, continue generating the trial solution. Usually, after a specified number of algorithm iterations $n_{1}$ is performed, the temperature will be decreased by the temperature reduction factor $R_{\mathrm{Te}}$, even if no improvement in the optimum takes place. The temperature should be cooled properly to guarantee the solution

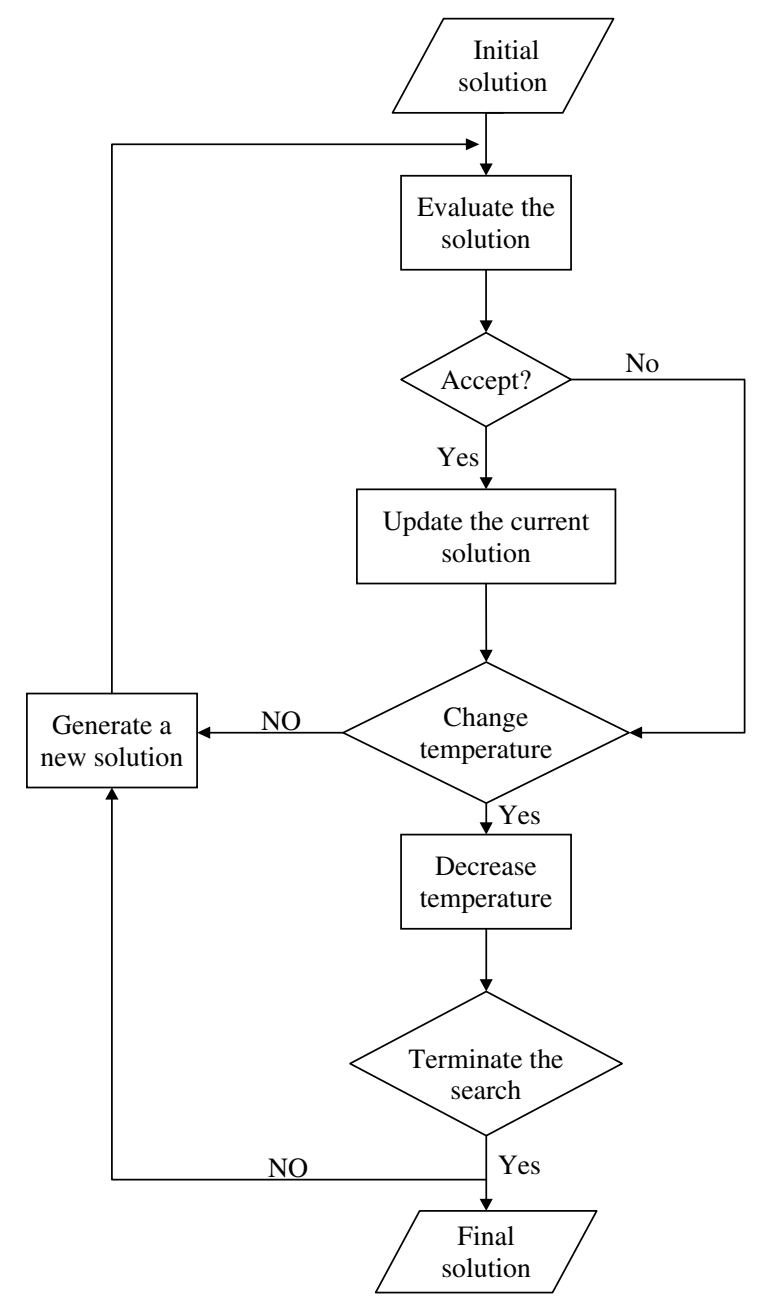

Figure 1. Flowchart of SA

obtained is indeed the global optimal solution (Zheng and Wang, 1996). The algorithm will be terminated when SA obtains the optimal solution or the solution obtained satisfies the stopping criteria. In general, the stopping criteria are defined to check whether the temperature or the difference between the optimal objective function value and those obtained in the current iteration reach the specified value or not.

\section{Genetic algorithms}

The GA, developed by Holland and his students during the period of 1960-1980, is an optimization algorithm inspired by both natural selection and natural genetics (Holland, 1975). Based on the theory of evolution, the better filial generation in the GA will survive and generate the next generation. Naturally, the best generation will have a superior presentation to adjust with the conditions. Rather than starting from a single point within a search space, the GA initiates a population of guesses. Therefore, the GA can be applied to an extremely wide range of optimization problems. There are five major steps required in a GA: encoding, decoding, selection, crossover, and mutation. In the encoding step, the initial guess of each unknown is converted to a binary string, called a substring, of length $l_{\text {id }}$. The total string length 
$T L$ is the summation of all the lengths of the substrings. Each string can be viewed as a simple data structure.

A group of initial guesses is required at the start of the GA optimization. A common way of generating the initial guesses is to use a random number generator. After generating the initial population of guesses, the decoding operator is then employed to decode each binary string to an integer or a real number to represent the unknown. Then these values are substituted into an objective function to calculate the fitness. Next, the other operations, selection, crossover, and mutation, will launch into this algorithm. The aim of the selection procedure is to reproduce a greater number of copies which have high fitness values. Poorer individuals are weeded out and better-performing individuals have a greater than average chance of promoting the information they contain within the next generation.

In the selection procedure, the proper individuals, based on those objective function values, will be put into a mating pool to reproduce offspring. Following the selection procedure, two new individuals (children) will be reproduced from two existing individuals (parents). Roughly, the individuals with a higher value have a higher probability of participating in reproduction and hence of contributing one or more offspring to the next generation. In general, the pair of individuals selected will or will not undergo crossover with a probability $P_{c}$. Typical values of $P_{c}$ range from 0.4 to 0.9 (Coley, 1999). There are three kinds of crossover method commonly applied: one-point crossover, two-point crossover, and homogeneous crossover (Coley, 1999). Mutation is used to change the value of single bit randomly within an

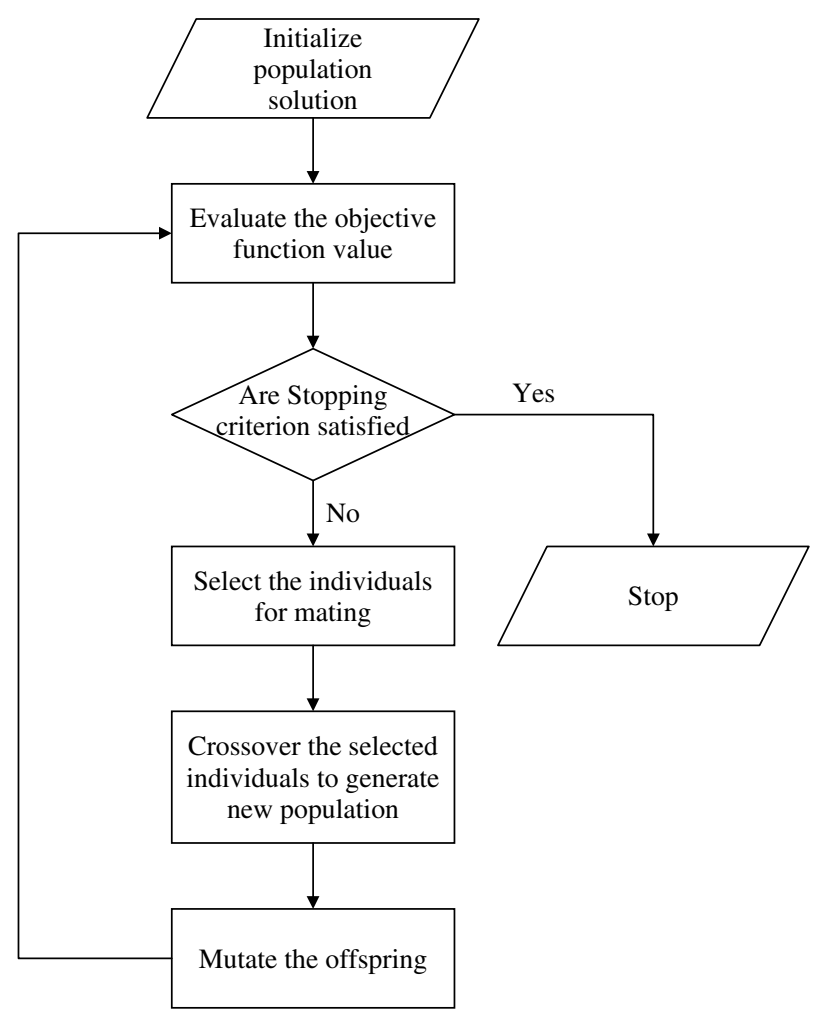

Figure 2. Flowchart of the GA individual string with a specified rate $P_{\mathrm{m}}$, the probability of mutation. Coley (1999) suggested using $P_{\mathrm{m}}$ of being $1 / T L$, or typically of the order $0 \cdot 001$, i.e. one bit in every thousand will be mutated.

Figure 2 demonstrates the flowchart of a GA. After giving the initial guesses, the trial solution selected, determined by the fitness function value, will get into the crossover step. In addition to the crossover step, each bit in the newborn trial solution, the binary string, will or will not mutate the value depending on the mutation probability. Finally, the algorithm is terminated when the solution or the number of iterations satisfies the stopping criteria.

\section{APPLICATION OF GLOBAL OPTIMIZATION METHODS}

This section illustrates how these two global optimization methods, the GA and SA, are coupled with a three- or four-parameter model to identify leaky aquifer parameters.

\section{Three-parameter model}

Hantush and Jacob's model describing the drawdown within a leaky aquifer in response to the pumping as a function of radial distance and time is (Hantush and Jacob, 1955; Batu, 1998)

$$
s=\frac{Q}{4 \pi T} W\left(u, \frac{r}{B}\right)
$$

where $B$ is the leakage factor and is defined as $\sqrt{\left(K^{\prime} / b^{\prime}\right) / T}$, where $K^{\prime}$ is the vertical conductivity of a leaky confining bed, $b^{\prime}$ is the thickness of aquitard, $r / B=L$ is the leakage coefficient, $r$ is the distance between the pumping well and observing well, $u$ is a dimensionless variable defined as $r^{2} S / 4 T t$, where $t$ is the pumping time, $W(u, r / B)$ is the leaky well function, $s$ is drawdown, and $Q$ is the pumping rate. Note that the typical values of $T, S$, and $B$ are between 0 and $3000 \mathrm{~m}^{2}$ day $^{-1}$, between $10^{-3}$ and $10^{-5}$, and between 0 and $1000 \mathrm{~m}$ respectively.

The leaky well function $W(u, r / B)$ may be expressed as

$$
W\left(u, \frac{r}{B}\right)=\int_{u}^{\infty} \frac{1}{y} \exp \left[-y-\frac{(r / B)^{2}}{4 y}\right] \mathrm{d} y
$$

where $y$ is a dummy variable. Since the right-hand side of Equation (6) is in integral form, a numerical approach may be required to evaluate the integration. Both the Laguerre quadrature formula and Gauss quadrature formula (Carnahan et al., 1969) can be employed to evaluate the values of leaky well function with an accuracy to the fourth decimal place.

\section{Four-parameter model}

The proposed global optimization methods are also applied along with Neuman and Witherspoon's (1969) 
model for analysing the leaky aquifer considering the storage effect in the confining layer. Given the initial estimates of the parameters, four best-fit parameters are obtained when the convergence criteria are met.

Neuman and Witherspoon (1969) presented a closed-form solution for the problem of flow to a well in a confined infinite radial system composed of two aquifers that are separated by an aquitard. Differing from Hantush and Jacob's (1955) work, Neuman and Witherspoon's model considers the effect of aquitard storage and drawdown in the unpumped aquifer. Their solution may be written as

$$
s=\frac{Q}{2 \pi T} \int_{0}^{\infty} \frac{1}{y}\left[1-\exp \left(-y^{2} \bar{t}_{\mathrm{D}}\right)\right] J_{0}[w(y)] \mathrm{d} y
$$

where $\bar{t}_{\mathrm{D}}=t_{\mathrm{D}} L^{2} / 16 \psi^{2}, t_{\mathrm{D}}=T t / r^{2} S, L=r / B, \psi=\beta / L$, $B=\sqrt{T b^{\prime} / K}, \beta=r \sqrt{S^{\prime}} / 4 B \sqrt{S}, J_{0}[w(y)]$ is the Bessel function of the first kind and zero order, and $w^{2}(y)=$ $L^{2} y^{2} / 16 \psi^{2}-L^{2} y \cot y$. The typical value of $\psi$ is between $10^{-3}$ and $10^{-5}$. Note that Equation (7) is valid for all values of time interval, and the Bessel function $J_{0}[w(y)]$ must be set to zero when $w^{2}(y)<0$.

\section{Objective functions for single-well and multiple-well data analyses}

The aquifer parameters are estimated based on either the three-parameter or four-parameter leaky aquifer model when finding the least sum of square errors between the observed and predicted drawdowns. Therefore, the objective function is defined as

$$
\text { Minimize } \sum_{i=1}^{n}\left(O h_{i}-P h_{i}\right)^{2}
$$

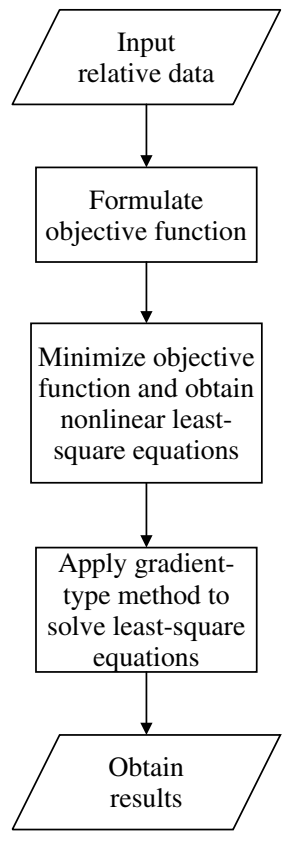

(a)

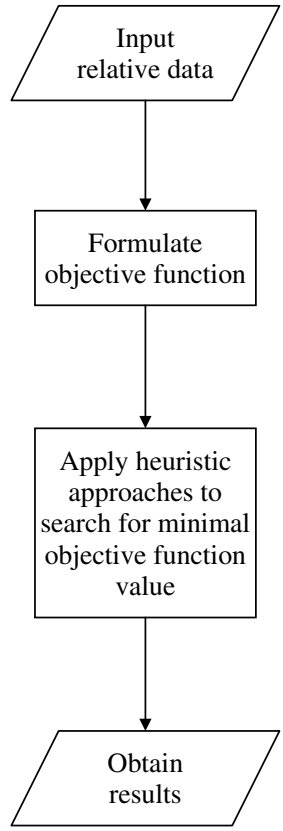

(b)
Figure 3. The procedure of the parameter identification: (a) gradient-type approach; (b) global optimization approach where $O h_{i}$ and $P h_{i}$ are respectively the observed and predicted drawdowns at different time steps and $n$ is the total number of data.

The aquifer parameter identification procedures for the gradient-type approach and global optimization approach are respectively demonstrated in Figure $3 \mathrm{a}$ and $\mathrm{b}$. The initial guesses are user defined in SA and generated by the random generator in the GA. After the initial guesses are given, the predicted drawdown is calculated using Equation (5) or (7). The most likely optimal solutions (trial solutions) will continue generating and improving based on the information of the objective function value. SA or the GA will terminate when the objective function value meets the specified stopping criteria.

\section{Error criteria}

To assess the accuracy of the estimated parameters, two error criteria, mean error ME and standard error of estimate SEE, are used to calculate the errors between the observed and predicted drawdowns in this study (Yeh, 1987; Yeh and Han, 1989). Note that the SEE is defined as the sum of the square difference between the observed and predicted drawdowns divided by $(n-p)$, where $p$ is the number of estimated parameters. Thus, the SEE value accounts for the number of target parameters. In reality, the more the target parameters are considered, the larger the SEE values will be when using different aquifer models to analyse the same drawdown data set.

\section{RESULTS AND DISCUSSION}

Table I lists the observed drawdown data, which are obtained from a test with three monitoring wells reported in Cooper (1963) and cited by Lohman (1972) for parameter identification using the three-parameter model. The distances between the pumping well and observation wells 1, 2, and 3 are $30.48 \mathrm{~m}, 152.4 \mathrm{~m}$, and $304.8 \mathrm{~m}$ respectively. The pumping rate $Q$ and total pumping time are $5450.98 \mathrm{~m}^{3} \mathrm{day}^{-1}$ and $1000 \mathrm{~min}$ respectively. In the four-parameter model, the time-drawdown data

Table I. Cooper's data obtained from three observation wells

\begin{tabular}{|c|c|c|c|}
\hline \multirow[t]{2}{*}{ Time (min) } & \multicolumn{3}{|c|}{ Observed drawdown (m) } \\
\hline & Well 1 & Well 2 & Well 3 \\
\hline $0 \cdot 2$ & 0.536 & $0 \cdot 003$ & 0 \\
\hline $0 \cdot 5$ & $0 \cdot 838$ & $0 \cdot 043$ & 0 \\
\hline 1 & 1.094 & $0 \cdot 137$ & 0.006 \\
\hline 2 & 1.298 & $0 \cdot 284$ & 0.043 \\
\hline 5 & 1.609 & 0.536 & 0.168 \\
\hline 10 & 1.798 & 0.713 & $0 \cdot 302$ \\
\hline 20 & 1.972 & 0.869 & 0.445 \\
\hline 50 & $2 \cdot 109$ & 1.009 & 0.594 \\
\hline 100 & $2 \cdot 167$ & 1.067 & 0.640 \\
\hline 200 & $2 \cdot 195$ & 1.07 & 0.643 \\
\hline 500 & $2 \cdot 198$ & 1.073 & 0.643 \\
\hline 1000 & $2 \cdot 198$ & 1.073 & 0.643 \\
\hline
\end{tabular}
(Cooper, 1963) 
Table II. Sridharan's pumping test data for four-parameter model (Sridharan et al., 1987)

\begin{tabular}{rl}
\hline Time (min) & Observed drawdown (m) \\
\hline 5 & $0 \cdot 3$ \\
28 & 0.95 \\
41 & $1 \cdot 1$ \\
60 & $1 \cdot 25$ \\
75 & $1 \cdot 34$ \\
250 & 1.75 \\
500 & 1.9 \\
700 & 1.95 \\
970 & 1.98 \\
1000 & 1.99 \\
1200 & 1.99 \\
\hline
\end{tabular}

are taken from Sridharan et al. (1987) and listed in Table II. The distance between the pumping well and the observation well is $29.0 \mathrm{~m}$ and the pumping rate $Q$ is $136 \cdot 26 \mathrm{~m}^{3}$ day $^{-1}$.

\section{Three-parameter model}

The upper and lower bounds for parameters estimated by SA and the GA when analysing field data using the three-parameter model are given in Table III. The initial temperature, the reduction factor $R_{\mathrm{t}}$, and the number

Table III. The upper and lower bounds for parameters used in $\mathrm{SA}$ and the GA in the data analysis of the three-parameter model

\begin{tabular}{|c|c|c|c|c|c|c|}
\hline \multirow[t]{2}{*}{ Method } & \multicolumn{2}{|c|}{$T\left(\mathrm{~m}^{2} \mathrm{day}^{-1}\right)$} & \multicolumn{2}{|c|}{$S$} & \multicolumn{2}{|c|}{$B(\mathrm{~m})$} \\
\hline & $\begin{array}{l}\text { Upper } \\
\text { bound }\end{array}$ & $\begin{array}{l}\text { Lower } \\
\text { bound }\end{array}$ & $\begin{array}{l}\text { Upper } \\
\text { bound }\end{array}$ & $\begin{array}{l}\text { Lower } \\
\text { bound }\end{array}$ & $\begin{array}{l}\text { Upper } \\
\text { bound }\end{array}$ & $\begin{array}{l}\text { Lower } \\
\text { bound }\end{array}$ \\
\hline SA & 3000 & 0 & $10^{-3}$ & $10^{-5}$ & 1000 & 0 \\
\hline GA & 3000 & 0 & $10^{-3}$ & $10^{-5}$ & 1000 & 0 \\
\hline
\end{tabular}

of algorithm iterations $n_{l}$ of SA are 10, 0.75, and 100 respectively. SA will be terminated if the absolute differences between the two successive values of the optimal objective function are all less than $10^{-6}$ within four iterations. The algorithmic parameters in the GA include the length of substrings $l_{1}, l_{2}$, and $l_{3}$ and are given as 11,10 , and 10 respectively. The crossover rate $P_{\mathrm{c}}$ and mutation rate $P_{\mathrm{m}}$ are 0.8 and 0.005 respectively. The population size is given as 3000 , and the stopping criterion is 500 generations at most.

The results obtained from SA and the GA are compared with those obtained from the EKF and NLN methods (Yeh and Huang, 2005) and are listed in Table IV. The

Table IV. Comparison of results from three-parameter model when using NLN, EKF, and global optimization methods to analyse Cooper's (1963) data

\begin{tabular}{|c|c|c|c|c|c|}
\hline \multirow{2}{*}{$\begin{array}{l}\text { Observation } \\
\text { well }\end{array}$} & \multicolumn{3}{|c|}{ Estimated parameters } & \multicolumn{2}{|c|}{ Errors } \\
\hline & $\begin{array}{l}T\left(\mathrm{~m}^{2}\right. \\
\left.\text { day }^{-1}\right)\end{array}$ & $\begin{array}{c}S \\
\left(\times 10^{-4}\right)\end{array}$ & $\begin{array}{c}B \\
(\mathrm{~m})\end{array}$ & $\begin{array}{c}\mathrm{ME} \\
\left(\times 10^{-4}\right)\end{array}$ & $\begin{array}{c}\mathrm{SEE} \\
\left(\times 10^{-3}\right)\end{array}$ \\
\hline \multicolumn{6}{|c|}{ SA method } \\
\hline 1 & $1239 \cdot 4$ & 0.98 & $617 \cdot 6$ & $3 \cdot 50$ & $13 \cdot 3$ \\
\hline 2 & $1243 \cdot 4$ & 0.97 & $605 \cdot 3$ & $-0 \cdot 385$ & 5.73 \\
\hline 3 & $1221 \cdot 2$ & 1.01 & 597.4 & -1.54 & 3.43 \\
\hline \multicolumn{6}{|c|}{ GA method } \\
\hline 1 & $1242 \cdot 2$ & 0.97 & 622.0 & $6 \cdot 26$ & $13 \cdot 4$ \\
\hline 2 & $1210 \cdot 9$ & 0.98 & 579.5 & $7 \cdot 29$ & $6 \cdot 33$ \\
\hline 3 & $1225 \cdot 6$ & 1.03 & $601 \cdot 2$ & $-10 \cdot 8$ & 4.02 \\
\hline \multicolumn{6}{|c|}{ EKF method } \\
\hline 1 & $1257 \cdot 9$ & 0.909 & 632.4 & $-6 \cdot 53$ & 19.9 \\
\hline 2 & $1311 \cdot 4$ & 0.929 & 668.4 & $37 \cdot 2$ & $8 \cdot 62$ \\
\hline 3 & 1228 & $1 \cdot 0$ & $600 \cdot 0$ & -2.44 & 4.09 \\
\hline \multicolumn{6}{|c|}{ NLN method } \\
\hline 1 & $1239 \cdot 1$ & 0.98 & $609 \cdot 6$ & $-1 \cdot 10$ & $13 \cdot 3$ \\
\hline 2 & $1242 \cdot 1$ & 0.98 & $603 \cdot 5$ & 4.98 & 5.69 \\
\hline 3 & $1215 \cdot 2$ & 0.97 & 594.8 & -1.90 & $3 \cdot 72$ \\
\hline
\end{tabular}

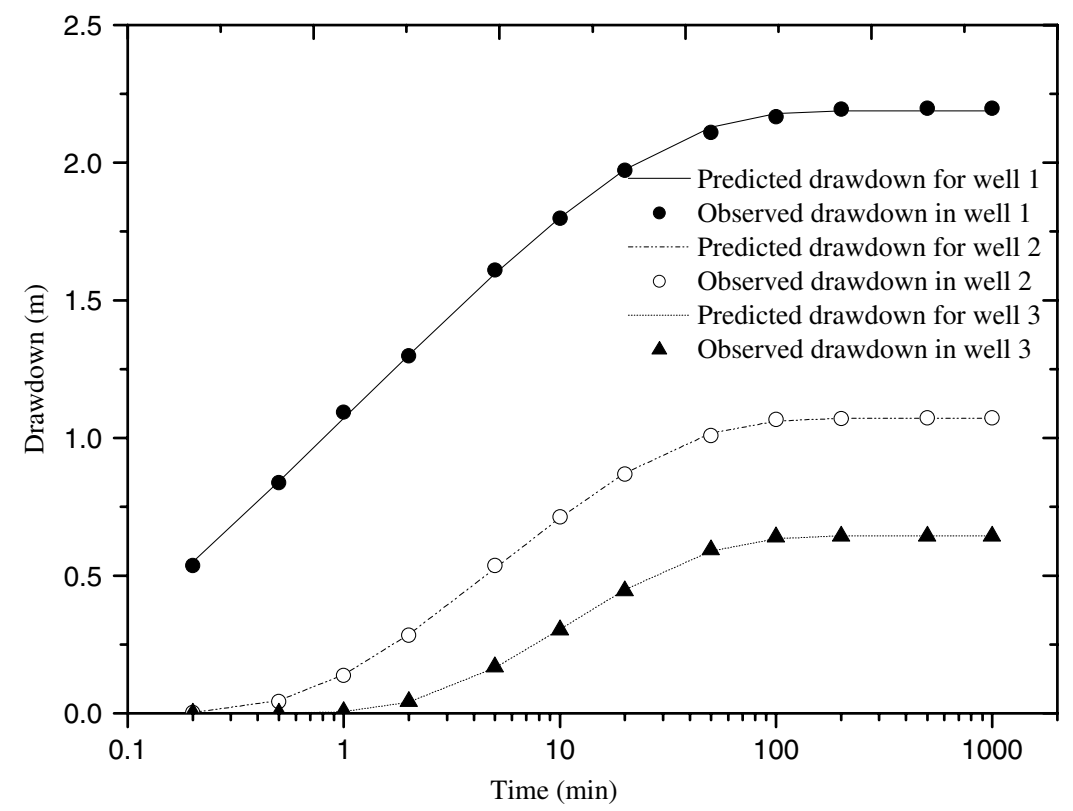

Figure 4. The predicted drawdowns obtained from SA and the pumping test data for the observation wells in the leaky aquifer without considering storage effect 


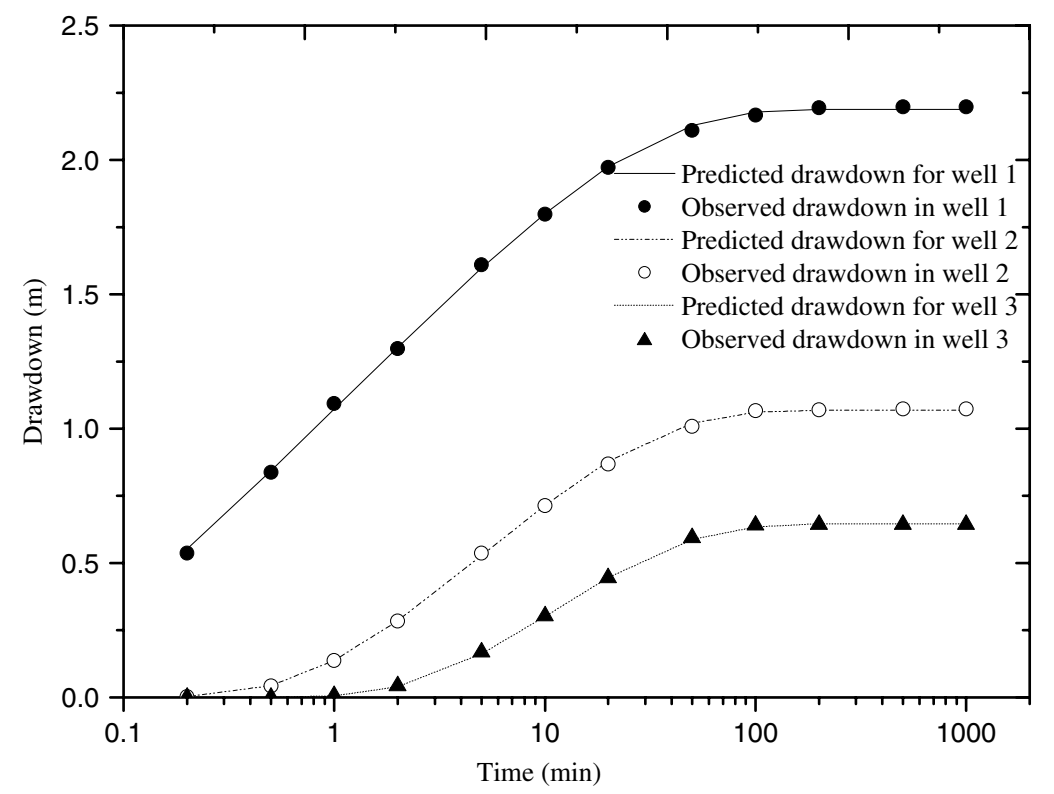

Figure 5. The predicted drawdowns obtained from the GA and the pumping test data for the observation wells in the leaky aquifer without considering storage effect

Table V. The upper and lower bounds for parameters used in SA and the GA in the data analysis of the four-parameter model

\begin{tabular}{|c|c|c|c|c|c|c|c|c|}
\hline \multirow[t]{2}{*}{ Method } & \multicolumn{2}{|c|}{$T\left(\mathrm{~m}^{2} \mathrm{day}^{-1}\right)$} & \multicolumn{2}{|c|}{$S$} & \multicolumn{2}{|c|}{$B(\mathrm{~m})$} & \multicolumn{2}{|c|}{$\psi$} \\
\hline & $\begin{array}{l}\text { Upper } \\
\text { bound }\end{array}$ & $\begin{array}{l}\text { Lower } \\
\text { bound }\end{array}$ & $\begin{array}{l}\text { Upper } \\
\text { bound }\end{array}$ & $\begin{array}{l}\text { Lower } \\
\text { bound }\end{array}$ & $\begin{array}{l}\text { Upper } \\
\text { bound }\end{array}$ & $\begin{array}{l}\text { Lower } \\
\text { bound }\end{array}$ & $\begin{array}{l}\text { Upper } \\
\text { bound }\end{array}$ & $\begin{array}{l}\text { Lower } \\
\text { bound }\end{array}$ \\
\hline SA & 3000 & 0 & $10^{-3}$ & $10^{-5}$ & 1000 & 0 & $10^{-3}$ & $10^{-5}$ \\
\hline GA & 3000 & 0 & $10^{-3}$ & $10^{-5}$ & 1000 & 0 & $10^{-3}$ & $10^{-5}$ \\
\hline
\end{tabular}

estimated $T$ for drawdown data obtained from those three wells by the proposed methods, EKF, and NLN range from 1200 to $1300 \mathrm{~m}^{2}$ day $^{-1}$. The estimated $S$ ranges from $9.7 \times 10^{-5}$ to $1.0 \times 10^{-4}$ and the estimated leakage factor $B$ ranges from 579.5 to 668.4 . These results indicate that the study site is close to the homogeneous aquifer system. Figures 4 and 5 show the observed drawdowns measured from those three wells and the predicted drawdowns generated by the Hantush and Jacob model with those parameters obtained from SA and the GA. Apparently, the predicted drawdowns quite suitably fit the pumping test data, as indicated in these two figures.

\section{Four-parameter model}

In the data analysis of the four-parameter model, the upper and lower bounds for parameters estimated by SA and the GA are given in the Table V. The algorithmic parameters in the GA include the lengths of substrings $l_{1}, l_{2}, l_{3}$, and $l_{4}$ and are given as $10,10,9$, and 10 respectively. Parameters $P_{\mathrm{c}}$ and $P_{\mathrm{m}}$ are the same as those given in the three-parameter model; the population size is given as 4000 and the stopping criterion herein is given as 500 generations at most.

The initial temperature is 10 , the reduction factor $R_{\mathrm{t}}$ is $0 \cdot 75$, and number of algorithm iterations $n_{l}$ is 100 . Notice that the identification procedure of $\mathrm{SA}$ is terminated when the absolute differences between the two successive values of the optimal objective function are all less than $10^{-6}$ within four iterations.

The results of estimation for parameters $T, S, L$, and $\psi$ are listed in Table VI. The estimated parameters obtained from SA and the GA almost agree with those obtained from NLN (Yeh and Huang, 2005), as indicated in Table VI. Figure 6 shows the observed drawdowns and the predicted drawdowns estimated by the Neuman and Witherspoon model and those parameters obtained from $\mathrm{SA}$ and the GA. Obviously, the differences between the predicated and observed drawdowns are extremely small.

\section{The key parameters of simulated annealing and the genetic algorithm}

The algorithmic parameters of SA or the GA are very critical in the searching procedures. Zheng and Wang (1996) suggested that the temperature reduction should be given properly to guarantee the results obtained are the global optimum. The population size in the GA controls the scale of the searching domain. To examine the robustness of applying the proposed approach in aquifer parameter identification, different key parameters of SA and the GA are assigned. The initial temperature and $R_{\mathrm{t}}$ in SA and the population size in the GA are considered as the key parameters in this study. Sridharan et al.'s (1987) data are analysed using the four-parameter model with different key SA parameters. Similarly, different key 
Table VI. The estimated parameters and prediction errors when using the NLN, EKF, and global optimization methods to analyse Sridharan et al.'s (1987) data

\begin{tabular}{lccccrrr}
\hline Method & \multicolumn{9}{c}{ Estimated parameters } & & \multicolumn{2}{c}{ Errors } \\
\cline { 2 - 4 } & $T\left(\mathrm{~m}^{2} \mathrm{day}^{-1}\right)$ & $S\left(\times 10^{-4}\right)$ & $B(\mathrm{~m})$ & $\psi\left(\times 10^{-4}\right)$ & & ME $\left(\times 10^{-3}\right)$ & SEE $\left(\times 10^{-2}\right)$ \\
\hline SA & 23.4 & 1.64 & 218.045 & 9.04 & -1.81 & 1.02 \\
GA & 23.9 & 1.59 & 228.346 & 9.68 & -2.67 & 1.18 \\
EKF & 22.6 & 1.73 & 204.225 & 3.16 & 1.49 & 1.36 \\
NLN & 23.3 & 1.65 & 216.418 & 7.04 & -1.78 & 1.00 \\
\hline
\end{tabular}

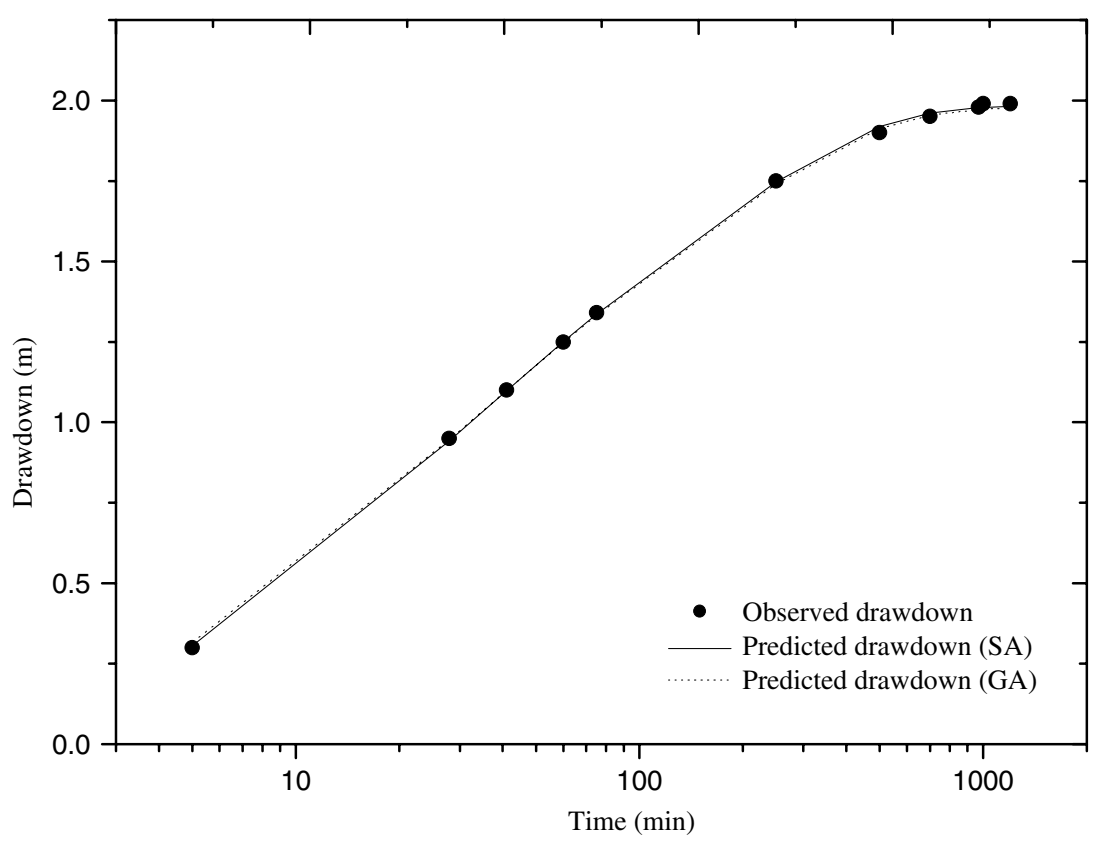

Figure 6. Comparison of the predicted drawdowns obtained from different global optimization methods and the pumping test data for the leaky aquifer with considering storage effect

GA parameters are chosen for analysing Cooper's (1963) data when using the three-parameter model.

Table VII lists the aquifer parameters obtained from SA with a different reduction factor $R_{\mathrm{t}}$. Those estimated parameters are almost the same except that the aquitard storage coefficient $\psi$ is slightly different. Table VIII lists the estimated results of different population sizes used in the GA for the drawdown data of the third observation well following 500 generation calculations. The aquifer parameters obtained by the GA when employing a small

Table VII. Estimated parameter by SA using different temperature reduction factors

\begin{tabular}{lcccc}
\hline $\begin{array}{l}\text { Temperature } \\
\text { reduction factor } R_{\mathrm{t}}\end{array}$ & \multicolumn{4}{c}{ Estimated parameters } \\
\cline { 2 - 5 } & $\begin{array}{c}T\left(\mathrm{~m}^{2}\right. \\
\left.\mathrm{day}^{-1}\right)\end{array}$ & $\begin{array}{c}S \\
\left(\times 10^{-4}\right)\end{array}$ & $\begin{array}{c}B \\
(\mathrm{~m})\end{array}$ & $\begin{array}{c}\psi \\
\left(\times 10^{-4}\right)\end{array}$ \\
\hline 0.90 & 23.36 & 1.64 & 218.05 & 9.06 \\
0.80 & 23.35 & 1.64 & 218.05 & 9.58 \\
0.75 & 23.36 & 1.64 & 218.05 & 9.04 \\
0.70 & 23.35 & 1.64 & 218.05 & 8.60 \\
0.60 & 23.35 & 1.64 & 218.05 & 9.57 \\
0.50 & 23.34 & 1.65 & 218.05 & 9.59 \\
0.30 & 23.35 & 1.64 & 218.05 & 9.58 \\
\hline
\end{tabular}

population size (300) are slightly different from those of a large population size (e.g. 1000 or/and 3000). However, the results obtained from a small population size can save about $90 \%$ of the computing time in obtaining results that are very close to the optimal solutions. Obviously, more accurate solutions require a larger population size and much more computing time. Figure 7 displays the evolution of the objective function value for different population sizes. The figure shows that the objective function value decreases rapidly in the early generations and very slowly in the latter generations, especially when the population size is 3000 . Note that the optimal solutions are obtained at 12,18 , and 20 generations as the population size is given as 3000, 1000, and 300 respectively. When a large population size is applied, more trial solutions will be produced and a better solution is more likely to be found. This fact also demonstrates that the GA is very robust in the early searching stage and the results converge to the optimal solution at a later searching stage.

If the initial guess values are far away from the target parameters, then gradient-type methods for solving the non-linear least-squares equations might give divergent results. This is the major disadvantage of employing 
Table VIII. Comparison of results for leaky aquifer without storage effect obtained by the GA using different population sizes

\begin{tabular}{lcccccr}
\hline Population size & $T\left(\mathrm{~m}^{2} \mathrm{day}^{-1}\right)$ & $S\left(\times 10^{-4}\right)$ & $B(\mathrm{~m})$ & ME $\left(\times 10^{-4}\right)$ & SEE $\left(\times 10^{-3}\right)$ & CPU time $(\mathrm{s})$ \\
\hline 300 & $1245 \cdot 61$ & 1.03 & 613.3 & 7.78 & 4.13 & $26 \cdot 90$ \\
1000 & 1225.59 & 1.03 & 601.2 & 9.76 & 4.02 & $100 \cdot 80$ \\
3000 & 1225.59 & 1.03 & 601.2 & 9.76 & 4.02 & $258 \cdot 70$
\end{tabular}

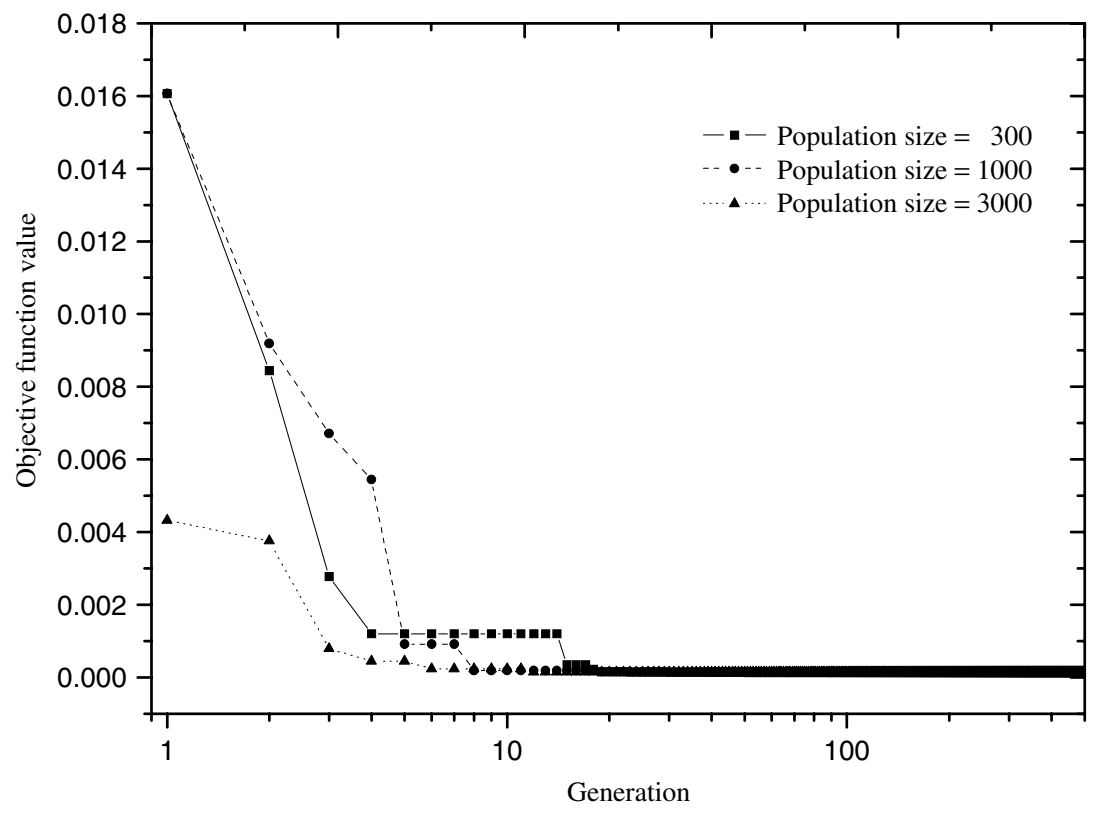

Figure 7. The objective function value versus generation for different population sizes

Table IX. Comparison of results in leaky aquifer considering storage effect when using different initial guesses for SA

\begin{tabular}{|c|c|c|c|c|c|c|c|c|}
\hline \multicolumn{4}{|c|}{ Initial guess } & \multicolumn{4}{|c|}{ Estimated parameters } & \multirow[t]{2}{*}{ Convergence or not? } \\
\hline$T\left(\mathrm{~m}^{2} \mathrm{day}^{-1}\right)$ & $S\left(\times 10^{-4}\right)$ & $L$ & $\psi\left(\times 10^{-4}\right)$ & $T\left(\mathrm{~m}^{2} \mathrm{day}^{-1}\right)$ & $S\left(\times 10^{-4}\right)$ & $B(\mathrm{~m})$ & $\psi\left(\times 10^{-4}\right)$ & \\
\hline \multirow[t]{5}{*}{$0 \cdot 0$} & $0 \cdot 1$ & 2.5 & $1 \cdot 0$ & $23 \cdot 3$ & 1.64 & $218 \cdot 05$ & $9 \cdot 58$ & Yes \\
\hline & $1 \cdot 0$ & & & $23 \cdot 3$ & 1.64 & $218 \cdot 05$ & 9.58 & Yes \\
\hline & $10 \cdot 0$ & & & $23 \cdot 3$ & 1.64 & $218 \cdot 05$ & $9 \cdot 57$ & Yes \\
\hline & $0 \cdot 1$ & 0.00 & 0.1 & $23 \cdot 3$ & 1.64 & 218.05 & 9.57 & Yes \\
\hline & & $0 \cdot 10$ & $10 \cdot 0$ & $23 \cdot 3$ & 1.64 & 218.05 & 9.57 & Yes \\
\hline \multirow[t]{4}{*}{$1000 \cdot 0$} & $0 \cdot 1$ & $2 \cdot 5$ & 1.0 & $23 \cdot 3$ & 1.64 & 218.05 & 9.59 & Yes \\
\hline & $1 \cdot 0$ & & & $23 \cdot 3$ & 1.64 & $218 \cdot 05$ & $9 \cdot 59$ & Yes \\
\hline & $10 \cdot 0$ & & & $23 \cdot 4$ & 1.64 & $218 \cdot 05$ & 9.57 & Yes \\
\hline & $1 \cdot 0$ & $0 \cdot 10$ & $10 \cdot 0$ & $23 \cdot 4$ & 1.64 & $218 \cdot 05$ & 9.04 & Yes \\
\hline \multirow[t]{5}{*}{$3000 \cdot 0$} & $0 \cdot 1$ & $2 \cdot 5$ & $1 \cdot 0$ & $23 \cdot 3$ & 1.64 & $218 \cdot 05$ & $9 \cdot 59$ & Yes \\
\hline & $1 \cdot 0$ & & & $23 \cdot 4$ & $1 \cdot 64$ & $218 \cdot 05$ & $9 \cdot 57$ & Yes \\
\hline & $10 \cdot 0$ & & & $23 \cdot 4$ & $1 \cdot 64$ & $218 \cdot 05$ & $9 \cdot 57$ & Yes \\
\hline & $10 \cdot 0$ & $5 \cdot 00$ & $10 \cdot 0$ & $23 \cdot 3$ & 1.64 & $218 \cdot 05$ & 9.58 & Yes \\
\hline & & 0.00 & $0 \cdot 1$ & $23 \cdot 4$ & 1.64 & $218 \cdot 05$ & $9 \cdot 04$ & Yes \\
\hline
\end{tabular}

the NLN method in solving non-linear least-squares equations. Therefore, different initial guess values for SA and different random number seeds for the GA are chosen to examine the performance of SA and the GA in parameter identification. Table IX displays the parameters obtained by SA with different initial guesses. The estimated parameters are almost identical, even if the initial guesses differ by several orders of magnitude. These results indicate that SA can not only determine aquifer parameters successfully, but also give a consistent estimation when using different reductions in the temperature factor and initial guesses. Figure 8 indicates that the results and the searching progress in the GA are the same with different random number seeds. Notice that the random number generator used in the GA is taken from Press et al. (1992) and it generates random values between zero and one based on the seed user defined. 


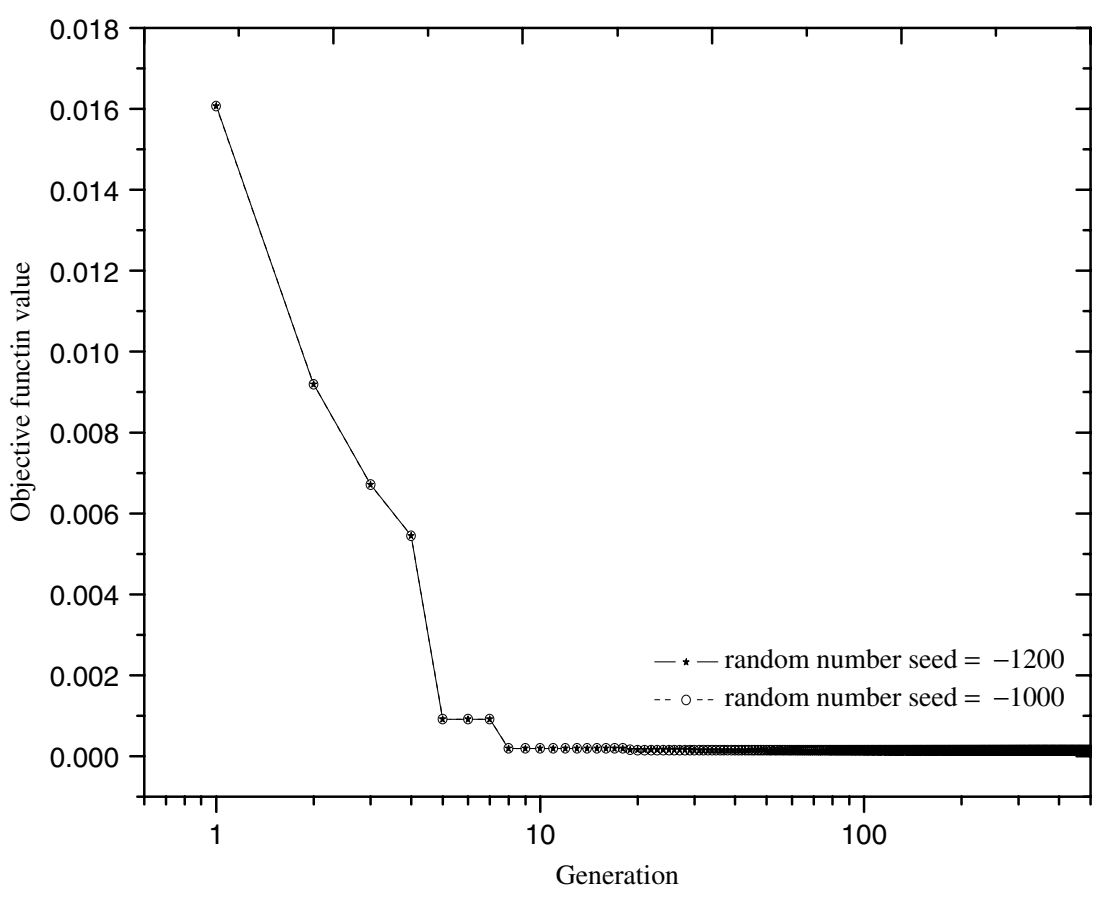

Figure 8 . The objective function value versus generation for different random number seeds

\section{CONCLUDING REMARKS}

A novel approach is developed based on global optimization methods such as SA or a GA and incorporated with aquifer drawdown equations to identify aquifer parameters of leaky aquifer systems. Two leaky aquifer models, one by Hantush and Jacob (1955) and the other by Neuman and Witherspoon (1969), are chosen to generate the drawdown data. This study employed SA and a GA to find the least sum of square errors between the observed drawdowns and the estimated drawdowns to identify the aquifer parameters. The Hantush and Jacob model contains three parameters: transmissivity, storage coefficient, and leakage factor. The Neuman and Witherspoon model has an additional parameter accounting for aquitard storativity. Three sets of drawdown data given by Cooper (1963) and the drawdown data given by Sridharan et al. (1987) were chosen for data analyses. The aquifer parameters obtained from SA or the GA agree suitably with those obtained from NLN or EKF coupled with the Hantush and Jacob model or the Neuman and Witherspoon model when analysing those available drawdown data.

The estimated parameters are almost identical for various initial guesses, which differ by several orders of magnitude. These results indicate that not only can SA determine aquifer parameters successfully, but it also gives consistent estimations when using different initial guesses. This is a significant advantage over the NLN and EKF approaches. In addition, different key SA and GA parameters are employed to analyse the aquifer parameters. The results indicate that the present approach always yields a good estimation for aquifer parameters when using different algorithmic parameters. In SA, the results obtained from random initial guesses and the different temperature reduction factors differ slightly from one other. Similarly, the differences in estimated parameters are negligible when varying the population size or changing the random number seed in the GA. In short, all the results of parameter estimations indicate that SA and the GA are very robust and give consistent results for handling the parameter identification problems.

\section{ACKNOWLEDGEMENTS}

This study was partly supported by the Taiwan National Science Council under grant NSC 93-2218-E009-056.

\section{REFERENCES}

Batu V. 1998. Aquifer Hydraulics. Wiley: New York.

Carnahan B, Luther HA, Wilkes JO. 1969. Applied Numerical Methods. Wiley: New York.

Coley DA. 1999. An Introduction to Genetic Algorithms for Scientists and Engineers. World Scientific.

Cooper HH. 1963. Type curves for nonsteady radial flow in an infinite leaky artesian aquifer. In Shortcuts and Special Problems in Aquifer Tests, Bentall R (compiler). US Geological Survey Water-Supply Paper 1545-C. USGS.

Cunha MDC, Sousa J. 1999. Water distribution network design optimization: simulated annealing approach. Journal of Water Resources Planning and Management, ASCE 125(4): 215-221.

Dawson KJ, Istok JD. 1991. Aquifer Testing: Design and Analysis of Pumping and Slug Tests. Lewis Publishers: Michigan.

Goffe WL, Ferrier G.D, Rogers J. 1994. Global optimization of statistical functions with simulated annealing. Journal of Econometrics. 60: $65-100$.

Hantush MS, Jacob CE. 1955. Non-steady radial flow in an infinite leaky aquifer. Transactions, American Geophysical Union 36: 95-100.

Holland J. 1975. Adaptation in Natural and Artificial Systems. University of Michigan Press.

Jacob CE. 1946. Radial flow in a leaky artesian aquifer. Transactions, American Geophysical Union 27: 198-208.

Kirkpatrick S, Gelatt Jr CD, Vecchi MP. 1983. Optimization by simulated annealing. Science 220(4598): 671-680. 
Lohman SW. 1972. Ground-water hydraulics. US Geological Survey Professional Paper $\mathbf{7 0 8}$

McKinney DC, Lin MD. 1994. Genetic algorithm solution of groundwater management models. Water Resources Research 30(6): 1897-1906.

Metropolis N, Rosenbluth AW. Rosenbluth MN, Teller AH, Teller E. 1953. Equation of state calculations by fast computing machines. Journal of Chemical Physics 21(6): 1087-1092.

Neuman SP, Witherspoon PA. 1969. Theory of flow in a confined two aquifer system. Water Resources Research 5(4): 803-816.

Pham DT, Karaboga D. 2000. Intelligent Optimisation Techniques. Springer: Great Britain.

Press WP, Teukolsky SA, Vetterling WT, Flannery BP. 1992. Numerical Recipes. Cambridge University Press.
Sridharan K, Ramaswamy R, Rao NSL. 1987. Identification of parameters in semiconfined aquifers. Journal of Hydrology 93: 163-173.

Yeh HD. 1987. Theis' solution by nonlinear least-squares and finitedifference Newton's method. Ground Water. 25: 710-715.

Yeh HD, Han HY. 1989. Numerical identification of parameters in leaky aquifers. Ground Water 27(5): 655-663.

Yeh HD, Huang YC. 2005. Parameter estimation for leaky aquifers using the extended Kalman filter with considering model and data measurement uncertainties, Journal of Hydrology 302: 28-45.

Zheng C, Wang P. 1996. Parameter structure identification using tabu search and simulated annealing. Advances in Water Resources 19(4): $215-224$. 